\title{
Phosphonium salts and aldehydes from the convenient, anhydrous reaction of aryl acetals and triphenylphosphine hydrobromide
}

\author{
Mani Ramanathan and Duen-Ren Hou* \\ Department of Chemistry, National Central University, 300 Jhong-Da Rd., Jhongli City, \\ Taoyuan, Taiwan \\ E-mail:drhou@ncu.edu.tw
}

\begin{abstract}
The reactions of aryl acetals/ketals and triphenylphosphine hydrobromide gave the corresponding aldehydes/ketones and alkyl phosphonium bromides. This reaction was applied to convert acetals/ketals to the corresponding aldehydes/ketones under an anhydrous and convenient condition $\left(50{ }^{\circ} \mathrm{C}, 5 \mathrm{~min}\right.$, up to $90 \%$ yield), and acid sensitive functional groups were compatible.
\end{abstract}

Keywords: Acetal, phosphonium salt, protecting group, anhydrous deprotection.

\section{Introduction}

Preparation and removal of acetals/ketals are often encountered in organic synthesis since the two functionalities are the primary protecting groups for aldehydes and ketones. ${ }^{1}$ The deprotection of acetals/ketals is usually achieved by acidic, aqueous hydrolysis. ${ }^{1,2}$ However, such conditions may cause problems for moisture- or acid-sensitive substrates. Several reagents, such as (trimethylsilyl)-bis(fluorosulfuryl)imide, ${ }^{3}$ dimethyldioxirane (DMDO), ${ }^{4}$ iodine, ${ }^{5}$ trimethylsilyl triflate, ${ }^{6}$ acetyl chloride/zinc chloride,${ }^{7}$ phosphorus triiodide, ${ }^{8}$ and photocleavage, ${ }^{9}$ have been developed to accomplish the deprotection under an anhydrous condition. We have found that the reaction of aryl acetals with the stable, non-hygroscopic, metal-free and commercially available salt, triphenylphosphine hydrobromide $\left(\mathrm{PPh}_{3} \cdot \mathrm{HBr}\right)^{10}$, provides the corresponding aldehydes/ketones and phosphonium salts in good yields. This paper reports our work to develop this reaction as a convenient and selective anhydrous deprotection method for aryl acetals and ketals (Equation 1). 


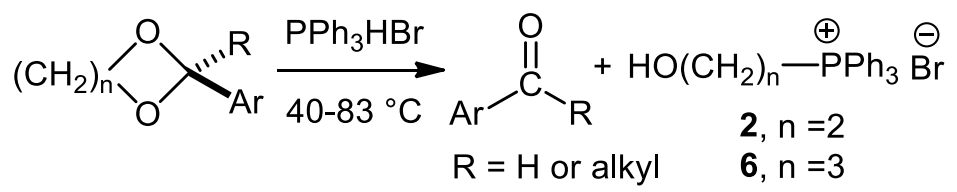

\section{Results and Discussion}

We previously reported that $\mathrm{PPh}_{3} \cdot \mathrm{HBr}$ was able to cleave benzyl ethers, ${ }^{11}$ which prompted us to apply this reagent to other functional groups. It was interesting to observe that aryl acetals/ketals were also susceptible to $\mathrm{PPh}_{3} \cdot \mathrm{HBr}$, but in a different reaction pathway. The reaction mixture of 2-phenyl-1,3-dioxolane (1) and $\mathrm{PPh}_{3} \cdot \mathrm{HBr}$, refluxed in dichloromethane for $3 \mathrm{~h}$, provided the two products: benzaldehyde (68\%) and (2-hydroxyethyl)triphenylphosphonium bromide (2, 73\%), which were isolated and characterized. The spectroscopic data $\left({ }^{1} \mathrm{H},{ }^{13} \mathrm{C},{ }^{31} \mathrm{P} \mathrm{NMR}\right)$ and mass analysis of this ionic product were consistent with those of (2hydroxyethyl)triphenylphosphonium bromide. ${ }^{12}$ The separation of the products could be easily achieved by adding diethyl ether to the concentrated product mixture, and the phosphonium salt precipitated.

To further improve the efficiency of this protocol, we applied microwave assisted heating and shortened the reaction time to $5 \mathrm{~min}$ at $50{ }^{\circ} \mathrm{C}$ in a sealed tube. Alternatively, conventional heating using 1,2-dichloroethane (bp $83{ }^{\circ} \mathrm{C}$ ) also reduced the reaction time to $30 \mathrm{~min}$. The results for the deprotection of some aryl acetals/ketals by $\mathrm{PPh}_{3} \cdot \mathrm{HBr}$ were summarized in Table 1 . The reaction of 2-methyl-2-phenyl-1,3-dioxolane (3) provided the same salt $\mathbf{2}$ and acetophenone, showing that this reaction pathway is general for both aryl acetals and ketals (entry 2).

The six-membered 2-phenyl-1,3-dioxane (4) generated (3-hydroxypropyl)triphenylphosphonium bromide $\left(6,{ }^{13} 84 \%\right.$, entry 3$)$. The acyclic acetal 5 gave ethyltriphenylphosphonium bromide ( $7,{ }^{14} 66 \%$, entry 4 ), in addition to the benzaldehyde. Both electron donating (entries 5, 6 and 9) and withdrawing groups (entries 7, 8, 10 and 11) on the aromatic ring were acceptable for this reaction, although the substrates with the electron withdrawing nitro group gave the lower yields. The non-reducing nature of $\mathrm{PPh}_{3} \cdot \mathrm{HBr}$ is beneficial for transformations involving the reducing agent sensitive compounds, such as those with nitro or bromo- substituents. 2,6Dichlorophenyl acetal 14 and naphthyl acetal 15 also underwent the reaction smoothly (entries 11 and 12). 
Table 1

Yield of aldehyde/ketone
$(\%)^{\mathrm{a}, \mathrm{b}}$

a $50^{\circ} \mathrm{C}, 5 \mathrm{~min}$.

${ }^{\mathrm{b}}$ Isolated yields after column chromatography.

${ }^{c}$ Yield of the corresponding phosphonium salt. 
The anhydrous reaction conditions and water free work-up procedures are favorable for the acetals/ketals bearing acid and moisture sensitive functional groups (Equation 2, Table 2). We found that common protecting groups for hydroxyls, such as the silyl groups (TBDPS and TBS, entries 1-2) and the substituted methyl ethers (MOM and Bn, entries 3-4) remained intact under this protocol. In contrast to the aryl acetal, the aliphatic, 1,3-dioxolane moiety of 20a was also compatible with triphenylphosphine hydrobromide (entry 5). The results from the substrates 19a and 20a demonstrated that the aryl acetals are far more reactive to $\mathrm{PPh}_{3} \cdot \mathrm{HBr}$ than the benzyl ether and the aliphatic acetal. This trend is consistent with the relative hydrolysis rate of aryl versus alkyl acetals, which suggests that the formation of the intermediate, oxocarbenium ion, is the rate determining step for both reactions (vide infra). ${ }^{15}$

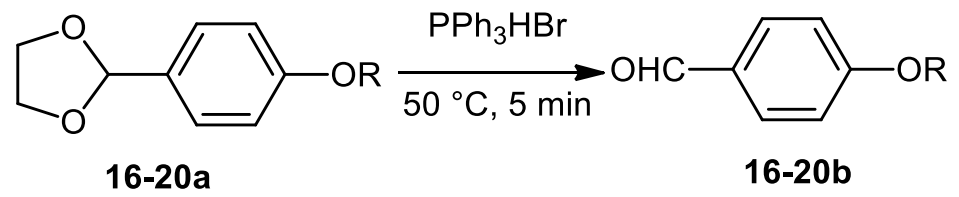

Table 2. Reactions of aryl acetals with protecting groups

\begin{tabular}{|c|c|c|c|}
\hline Entry & Reactant & Product & Yield $(\%)^{\mathrm{a}, \mathrm{b}}$ \\
\hline 1 & $\mathrm{R}=-\mathrm{Si}\left({ }^{t} \mathrm{Bu}\right) \mathrm{Ph}_{2}(\mathrm{TBDPS}), \mathbf{1 6 a}$ & $\mathbf{1 6 b}$ & $(89)$ \\
\hline 2 & $\mathrm{R}=-\mathrm{Si}\left({ }^{t} \mathrm{Bu}\right) \mathrm{Me}_{2}(\mathrm{TBS}), \mathbf{1 7 a}$ & $\mathbf{1 7 b}$ & 81 \\
\hline 3 & $\mathrm{R}=-\mathrm{CH}_{2} \mathrm{OCH}_{3}(\mathrm{MOM}), \mathbf{1 8 a}$ & $\mathbf{1 8 b}$ & 77 \\
\hline 4 & $\mathrm{R}=\mathrm{Bn}, \mathbf{1 9 a}$ & $\mathbf{1 9 b}$ & 68 \\
\hline 5 & $\mathrm{R}={ }^{-\left(\mathrm{CH}_{2}\right)_{4}-{ }_{\mathrm{O}}-\mathbf{2 0 a}}$ & $\mathbf{2 0 b}$ & 90 \\
\hline
\end{tabular}

a $50^{\circ} \mathrm{C}, 5 \mathrm{~min}$.

${ }^{\mathrm{b}}$ Isolated yields after column chromatography.

4-Substituted dioxolanes 21 and 22 were prepared to further explore this reaction. ${ }^{16}$ In addition to benzaldehyde, (2-hydroxypropyl)- and (2-hydroxy-2-phenylethyl)triphenylphosphonium salts $\mathbf{2 3}^{17}$ and $\mathbf{2 4}{ }^{18}$ (Equation 3) were produced. However, the other possible phosphonium salts, 25, with a primary hydroxyl group, were not observed (Scheme 1). These results indicated that triphenylphosphine only attacked the sterically less hindered primary carbon, rather than the secondary carbon or the $s p^{2}$ carbon, of the intermediate oxocarbenium ion. The latter pathway was observed in the reactions of the less hindered triethylphosphine. ${ }^{19}$ This unique property of $\mathrm{PPh}_{3} \cdot \mathrm{HBr}$ could be applied to prepare the corresponding phosphonium salts, 
such as $\mathbf{2 3}$ and $\mathbf{2 4}$, directly from the aryl acetals/ketals, rather than the sequence of deprotection, bromination and the formation of phosphonium salt, as shown in the reported syntheses. ${ }^{20}$

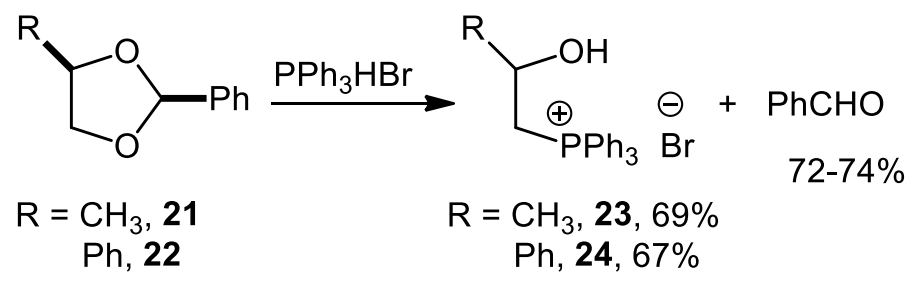<smiles>[R]C1COC(c2ccccc2)O1</smiles>

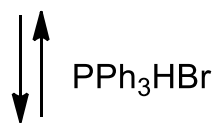

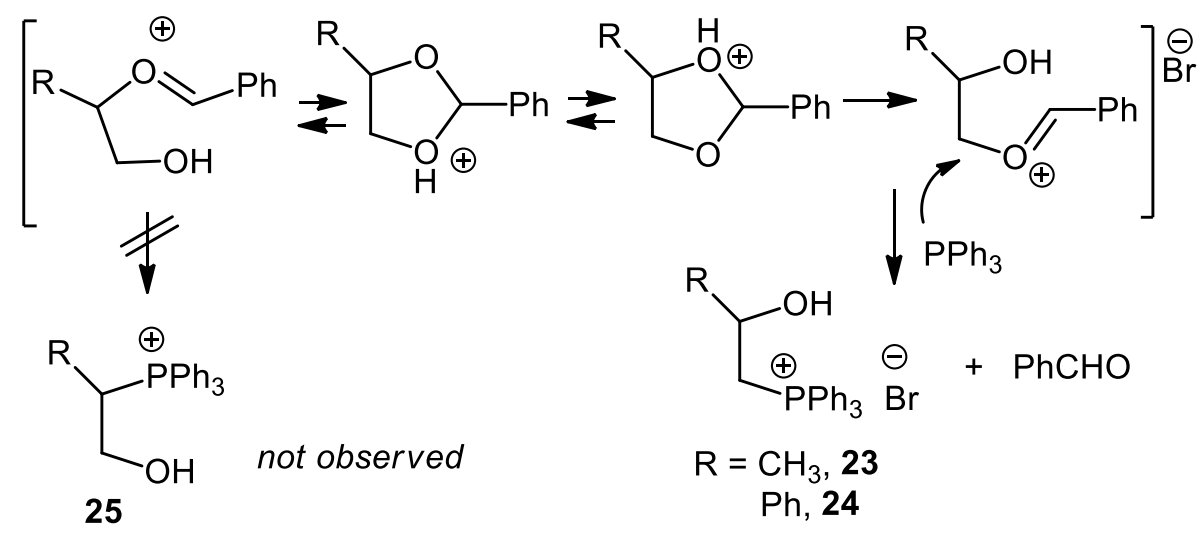

Scheme 1. Proposed reaction mechanism for the reactions of the aryl acetals and $\mathrm{PPh}_{3} \bullet \mathrm{HBr}$.

\section{Conclusions}

To our knowledge, the reaction of aryl acetals/ketals and triphenylphosphine hydrobromide was only briefly described. ${ }^{19}$ We developed this reaction as a convenient, deprotection method for aryl acetals/ketals and a direct preparation of the corresponding phosphonium salts. Its efficiency, mildness and anhydrous conditions could be useful for substrates with these concerns. The formation of the phosphonium salts is selective when the substituted dioxolanes are applied, and this process is different from that of triethylphosphine hydrobromide. 


\section{Experimental Section}

General. All purchased chemicals were used without further purification. THF was distilled from sodium benzophenone ketyl. ${ }^{1} \mathrm{H}$ and ${ }^{13} \mathrm{C}$ NMR spectra were obtained on $300 \mathrm{MHz}$ spectrometers and referenced to TMS or residual $\mathrm{CHCl}_{3}$. Analytical TLC was carried out using aluminum-backed $0.2 \mathrm{~mm}$ silica gel $60 \mathrm{~F} 254$ plates. Column chromatography was conducted using 230-400 mesh silica gel.

Compounds $1,{ }^{21} 3,{ }^{22} 4,{ }^{23} 5,{ }^{24} \mathbf{8},{ }^{24} \mathbf{1 0},{ }^{25} \mathbf{1 1},{ }^{26} \mathbf{1 2},{ }^{27} \mathbf{1 3}^{28}, \mathbf{1 5}^{29}, \mathbf{1 3 a}-\mathbf{b},{ }^{30} \mathbf{1 4 a - b},{ }^{23} \mathbf{1 5 b},{ }^{31} \mathbf{1 6} \mathbf{b},{ }^{32} \mathbf{1 8},{ }^{16}$ 19, ${ }^{16,33}$ were known, and were prepared according to the general procedure.

Standard procedure for the acetal deprotection. A solution of acetal $(0.66 \mathrm{mmol})$, triphenylphosphine hydrobromide $(0.73 \mathrm{mmol})$ in dry dichloromethane $(0.5 \mathrm{~mL})$ was placed in a reaction tube $\left(10 \mathrm{~mL}\right.$, for microwave reaction). The tube was capped and heated to $50{ }^{\circ} \mathrm{C}$ for 5 minutes in a microwave oven (CEM Discover). After cooling to room temperature, the reaction mixture was concentrated under vacuum. The alkyl phosphonium salts precipitated after adding diethyl ether, and the aldehyde was harvested after concentrating the filtrate. Alternatively, the separation of the two products could be achieved by flash column chromatography.

2-(2,6-Dichlorophenyl)-1,3-dioxolane (14). A mixture of 2,6-dichlorobenzaldehyde ( $0.5 \mathrm{~g}, 2.86$ $\mathrm{mmol})$, ethylene glycol $(0.8 \mathrm{~g}, 11.6 \mathrm{mmol})$ and $p-\mathrm{TsOH} . \mathrm{H}_{2} \mathrm{O}(54 \mathrm{mg}, 0.28 \mathrm{mmol})$ in dry toluene $(20 \mathrm{~mL})$ was equipped with a Dean-Stark apparatus and heated to reflux for $12 \mathrm{~h}$. After cooling the mixture to room temperature, added $10 \mathrm{~mL}$ of saturated aq. Sodium bicarbonate solution and extracted with ethyl acetate $(2 \times 25 \mathrm{~mL})$ combined organic layers were washed with brine $(20$ $\mathrm{mL}$ ), dried over sodium sulfate. Solvent was removed under reduced pressure and the residue was purified by silica gel column chromatography (5\%, ethyl acetate: hexanes, trace amount of triethylamine) to give the title compound $(0.38 \mathrm{~g}, 2.17 \mathrm{mmol}, 63 \%)$ as a colorless solid. Mp 44.5$46.0{ }^{\circ} \mathrm{C} ;{ }^{1} \mathrm{H}$ NMR $\left(300 \mathrm{MHz}, \mathrm{CDCl}_{3}\right) \delta 3.97-4.13(\mathrm{~m}, 4 \mathrm{H}), 6.45(\mathrm{~s}, 1 \mathrm{H}), 7.16-7.30(\mathrm{~m}, 3 \mathrm{H}) ;{ }^{13} \mathrm{C}$ $\operatorname{NMR}\left(75 \mathrm{MHz}, \mathrm{CDCl}_{3}\right) \delta 65.9,101.0,129.5,130.6,130.8,136.0$.

2-(4-(Methoxymethoxy) phenyl)-1,3-dioxolane (18a). A solution of 4-(1,3-dioxolan-2yl)phenol $(0.37 \mathrm{~g}, 2.23 \mathrm{mmol})$ and $N, N$-disopropylethylamine $(1.44 \mathrm{~g}, 1.9 \mathrm{~mL}, 11.13 \mathrm{mmol})$ in 5 $\mathrm{mL}$ dry dichloromethane was cooled to $0{ }^{\circ} \mathrm{C}$. A solution of methoxymethyl chloride $(0.36 \mathrm{~g}, 0.34$ $\mathrm{mL}, 4.45 \mathrm{mmol})$ and $N, N$-disopropylethylamine $(1.44 \mathrm{~g}, 1.9 \mathrm{~mL}, 11.13 \mathrm{mmol})$ in $5 \mathrm{~mL}$ dry dichloromethane was added slowly over $10 \mathrm{~min}$. The reaction mixture was warmed to room temperature and stirred for $6 \mathrm{~h}$. The reaction mixture was diluted with dichloromethane $(10 \mathrm{~mL})$, washed with saturated aqueous ammonium chloride $(5 \mathrm{~mL})$, saturated aqueous sodium bicarbonate $(5 \mathrm{~mL})$, water $(5 \mathrm{~mL})$, brine $(5 \mathrm{~mL})$. The combined organic layers were dried over sodium sulfate and the solvents were removed under reduced pressure. The crude mixture was purified by flash column chromotography (10\%, ethyl acetate: hexanes, trace amount of triethylamine). The title compound was isolated $(0.32 \mathrm{~g}, 1.52 \mathrm{mmol}, 68 \%)$ as colorless oil. ${ }^{1} \mathrm{H}$ NMR (300 MHz, $\left.\mathrm{CDCl}_{3}\right) \delta 3.37(\mathrm{~s}, 3 \mathrm{H}), 3.91-3.93(\mathrm{~m}, 2 \mathrm{H}), 4.00-4.05(\mathrm{~m}, 2 \mathrm{H}), 5.08(\mathrm{~s}, 2 \mathrm{H})$, $5.67(\mathrm{~s}, 1 \mathrm{H}), 6.95(\mathrm{~d}, 8.7 \mathrm{~Hz}, 2 \mathrm{H}), 7.31(\mathrm{~d}, 8.7 \mathrm{~Hz}, 2 \mathrm{H}) ;{ }^{13} \mathrm{C} \mathrm{NMR}\left(75 \mathrm{MHz}, \mathrm{CDCl}_{3}\right) \square \delta 55.9$, 
65.2, 94.2, 103.50, 115.9, 127.8, 131.2, 157.9. HRMS (FAB) calcd. for $\left[\mathrm{M}+\mathrm{H}^{+}\right]\left(\mathrm{C}_{11} \mathrm{H}_{15} \mathrm{O}_{4}\right)$, 211.0970; found 211.0970.

2-(4-(Benzyloxy) phenyl)-1,3-dioxolane (19a). A mixture of 4-(benzyloxy)benzaldehyde (0.5 g, $2.36 \mathrm{mmol})$, ethylene glycol $(0.9 \mathrm{~g}, 14.13 \mathrm{mmol})$ and $p$ - $\mathrm{TsOH} . \mathrm{H}_{2} \mathrm{O}(36 \mathrm{mg}, 0.19 \mathrm{mmol})$ in dry benzene $(15 \mathrm{~mL})$ was equipped with a Dean-Stark apparatus and heated to reflux for $24 \mathrm{~h}$. After cooling the mixture to room temperature, added $10 \mathrm{~mL}$ of saturated aq. Sodium bicarbonate solution and extracted with ethyl acetate $(2 \times 25 \mathrm{~mL})$ combined organic layers were washed with brine $(20 \mathrm{~mL})$, dried over sodium sulfate. Solvent was removed under reduced pressure and the residue was purified by silica gel column chromatography (20\%, ethyl acetate: hexanes, trace amount of triethylamine) to give the title compound $(0.40 \mathrm{~g}, 1.57 \mathrm{mmol}, 67 \%)$ as a colorless oil. ${ }^{1} \mathrm{H}$ NMR (300 MHz, $\left.\mathrm{CDCl}_{3}\right) \delta$ 3.97-4.13 (m, 4H), $5.06(\mathrm{~s}, 2 \mathrm{H}), 5.74(\mathrm{~s}, 1 \mathrm{H})$ 6.94-6.98 (m, 2H), 7.29-7.42 (m, 7H); $\left.{ }^{13} \mathrm{C} \mathrm{NMR} \mathrm{(75} \mathrm{MHz,} \mathrm{CDCl}_{3}\right) \delta$ 65.2, 70.0, 103.6, 115.2, 117.7, 127.4, 127.8, $127.9,128.5,136.8,159.5 .^{34}$

2-(4-(4-(1,3-Dioxolan-2-yl)butoxy)phenyl)-1,3-dioxolane (20a). A suspension of 4-(5oxopentyloxy)benzaldehyde $(0.5 \mathrm{~g}, 2.42 \mathrm{mmol})$, ethylene glycol $(0.9 \mathrm{~g}, 14.54 \mathrm{mmol})$ and $p$ $\mathrm{TsOH} \cdot \mathrm{H}_{2} \mathrm{O}$ (37 mg, $\left.0.19 \mathrm{mmol}\right)$ in dry toluene $(15 \mathrm{~mL})$ was equipped with a Dean-Stark apparatus and heated to reflux for $12 \mathrm{~h}$. After cooling the mixture to room temperature, added 10 $\mathrm{mL}$ of saturated aq. sodium bicarbonate solution and extracted with ethyl acetate $(2 \times 20 \mathrm{~mL})$, combined organic layers were washed with brine $(20 \mathrm{~mL})$, dried over sodium sulfate. Solvent was removed under reduced pressure and the residue was purified by silica gel column chromatography (20\%. ethyl acetate: hexanes, trace amount of triethylamine) to give the title compound (0.506 mg, $1.72 \mathrm{mmol}, 73 \%)$ as a colorless oil. ${ }^{1} \mathrm{H}$ NMR (300 $\left.\mathrm{MHz}, \mathrm{CDCl}_{3}\right) \delta 1.67$ $1.82(\mathrm{~m}, 6 \mathrm{H}), 3.81-4.09(\mathrm{~m}, 10 \mathrm{H}), 4.85(\mathrm{t}, 9 \mathrm{~Hz}, 3 \mathrm{H}), 5.71(\mathrm{~s}, 1 \mathrm{H}), 6.85(\mathrm{~d}, 8.7 \mathrm{~Hz}, 2 \mathrm{H}), 7.35(\mathrm{~d}$, $4.35 \mathrm{~Hz}, 2 \mathrm{H}) ;{ }^{13} \mathrm{C} \mathrm{NMR}\left(75 \mathrm{MHz}, \mathrm{CDCl}_{3}\right) \delta$ 20.5, 29.0, 33.4, 64.8, 65.2, 67.7, 103.7, 104.3, 114.2, 127.8, 129.7, 159.8. HRMS (FAB) calcd. for $\left[\mathrm{M}+\mathrm{H}^{+}\right]\left(\mathrm{C}_{16} \mathrm{H}_{23} \mathrm{O}_{5}\right), 295.1546$; found 295.1538 .

4-(4-(1,3-Dioxolan-2-yl)butoxy)benzaldehyde (20b). The standard procedure for the deprotection was followed. Starting with $\mathbf{2 0 a}(100.1 \mathrm{mg}, 0.34 \mathrm{mmol}), \mathbf{2 0 b}$ was isolated after silica gel column chromatography (20\% Ethyl acetate: Hexane, trace amount of triethylamine), as a colorless oil, (61.2 mg, $0.24 \mathrm{mmol}, 72 \%) .{ }^{1} \mathrm{H} \mathrm{NMR}\left(300 \mathrm{MHz}, \mathrm{CDCl}_{3}\right) \delta 1.72-1.89(\mathrm{~m}, 6 \mathrm{H})$, 3.81-4.05 (m, 6H), 4.86 (t, $4.8 \mathrm{~Hz}, 3 \mathrm{H}), 6.95$ (d, 8.7 Hz, 2H), 7.79 (d, 8.7 Hz, 2H), 9.85 (s, 1H); ${ }^{13} \mathrm{C}$ NMR (75 MHz, $\mathrm{CDCl}_{3}$ ), 20.5, 28.9, 33.4, 64.9, 68.1, 104.3, 114.7, 129.7, 132, 164.1, 190.8. HRMS (EI) calcd. for $\left[\mathrm{M}^{+}\right]\left(\mathrm{C}_{14} \mathrm{H}_{18} \mathrm{O}_{4}\right) 250.1205$, found 250.1206 .

2,4-Diphenyl-1,3-dioxolane (22). To a solution of 1-phenylethane-1,2-diol (500 mg, $3.62 \mathrm{mmol}$ ) and benzaldehyde $(576 \mathrm{mg}, 5.42 \mathrm{mmol})$ in dry dichloromethane $(6 \mathrm{~mL})$ was added $p$ - $\mathrm{TsOH} \cdot \mathrm{H}_{2} \mathrm{O}$ (63 mg, $0.09 \mathrm{mmol}$ ). The reaction mixture was stirred at room temperature for $15 \mathrm{~h}$, then diluted with $10 \mathrm{~mL}$ dichloromethane and washed with $5 \mathrm{~mL}$ of saturated aqueous sodium bicarbonate solution, washed with $5 \mathrm{~mL}$ of brine solution. The organic layer was dried over sodium sulfate and the solvents were removed under reduced pressure. The residue was purified by flash column chromatography ( $2 \%$, ethylacetate: hexanes with trace amount of triethylamine) to give 
the title compound (0.46 g, $2.0 \mathrm{mmol}, 44 \%){ }^{1} \mathrm{H} \mathrm{NMR}\left(300 \mathrm{MHz}, \mathrm{CDCl}_{3}\right) \delta 3.95(\mathrm{~m}, 1 \mathrm{H}), 4.38(\mathrm{t}$, $7.1 \mathrm{~Hz}, 1 \mathrm{H}), 5.21(\mathrm{t}, 7.1 \mathrm{~Hz}, 1 \mathrm{H}), 6.02(\mathrm{~s}, 1 \mathrm{H}), 7.32-7.62(\mathrm{~m}, 10 \mathrm{H}) ;{ }^{13} \mathrm{C} \mathrm{NMR}(75 \mathrm{MHz}$, $\left.\mathrm{CDCl}_{3}\right) \delta 72.2,78.8,104.6,126.4,126.7,128.2,128.4,128.5,129.3,137.4,139.0$.

\section{Acknowledgements}

This research was supported by the National Science Council (NSC 98-2119-M-008-001-MY3), Taiwan. We are grateful to Ms. Ping-Yu Lin at the Institute of Chemistry, Academia Sinica, and Valuable Instrument Center in National Central University for obtaining mass analysis.

\section{References}

1. (a) Wuts, P. G. M.; Greene, T. W. in Protective Groups in Organic Synthesis; 4th ed.; John Wiley \& Sons: New York, 2007; pp. 435-473.

(b) Kocienski, P. J. in Protecting Groups; 3rd ed.; Georg Thieme Verlag: Stuttgart, Germany, 2005.

(c) Smith, M. B. in Organic Synthesis; 3rd ed.; Wavefunction Inc.: Irvine, 2011; pp. 608-612.

2. Recent examples: (a) Ates, A.; Gautier, A.; Leroy, B.; Plancher, J.-M.; Quesnel, Y.; Vanherck, J.-C.; Marko, I. E. Tetrahedron 2003, 59, 8989.

(b) Robinson, M. W. C.; Graham, A. E. Tetrahedron Lett. 2007, 48, 4727.

http://dx.doi.org/10.1016/j.tetlet.2007.05.022

(c) Cayley, A. N.; Cox, R. J.; Ménard-Moyon, C.; Schmidta, J. P.; Taylor, R. J. K.

Tetrahedron Lett. 2007, 48, 6556.

http://dx.doi.org/10.1016/j.tetlet.2007.07.018

(d) Bailey, A. D.; Baru, A. R.; Tasche, K. K.; Mohan, R. S. Tetrahedron Lett. 2008, 49, 691.

(e) Olson, M. E.; Carolan, J. P.; Chiodo, M. V.; Lazzara, P. R. ; Mohan, R. S. Tetrahedron

Lett. 2010, 51, 3969.

(f) Golden, K. C.; Gregg, B. T.; Quinn, J. F. Tetrahedron Lett. 2010, 51, 4010.

http://dx.doi.org/10.1016/j.tetlet.2010.05.116

(g) Madabhushi, S.; Mallu, K. K. R.; Chinthala, N.; Beeram, C. R.; Vangipuram, V. S.

Tetrahedron Lett. 2012, 53, 697.

http://dx.doi.org/10.1016/j.tetlet.2011.11.135

(h) Ranu, B. C.; Jana, R.; Samanta, S. Adv. Synth. Catal. 2004, 346, 446.

http://dx.doi.org/10.1002/adsc.200303154

(i) Krishnaveni, N. S.; Surendra, K.; Reddy, M. A.; Nageswar, Y. V. D.; Rao, K. R. J. Org. Chem. 2003, 68, 2018.

http://dx.doi.org/10.1021/jo026482; PMid:12608827 
(j) Williams, D. B. G.; Cullen, A.; Fourie, A.; Henning, H.; Lawton, M.; Mommsen, W.; Nangu, P.; Parker, J.; Renison, A. Green Chem. 2010, 12, 1919. http://dx.doi.org/10.1039/c0gc00280a

(k) Chang, C.-C.; Liao, B.-S.; Liu, S.-T. Synlett 2007, 283.

(1) Mohammadpoor-Baltork, I.; Moghadam, M.; Tangestaninejad, S.; Mirkhani, V.; Mirjafari, A. Can. J. Chem. 2008, 86, 831. http://dx.doi.org/10.1139/v08-068

3. Kaur, G.; Trehan, A.; Trehan, S. J. Org. Chem. 1998, 63, 2365. http://dx.doi.org/10.1021/jo971756s

4. Mycock, D. K.; Sherlock, A. E.; Glossop, P. A.; Hayes, C. J. Tetrahedron Lett. 2008, 49, 6390. http://dx.doi.org/10.1016/j.tetlet.2008.08.062

5. Sun, J.; Dong, Y.; Cao, L.; Wang, X.; Wang, S.; Hu, Y. J. Org. Chem. 2004, 69, 8932. http://dx.doi.org/10.1021/jo0486239; PMid:15575776

6. Fujioka, H.; Minamitsuji, Y.; Kubo, O.; Senami, K.; Maegawa, T. Tetrahedron 2011, 67, 2949. http://dx.doi.org/10.1016/j.tet.2011.02.048

7. Chang, C.; Chua, K. C.; Yue, S. Syn. Commun. 1992, 22, 1217. http://dx.doi.org/10.1080/00397919208021108

8. Denis, J.-N.; Krief, A. Angew. Chem. Int. Ed. Engl. 1980, 19, 1006. http://dx.doi.org/10.1002/anie.198010061

9. (a) Yang, H.; Zhang, X.; Zhou, L.; Wang, P. J. Org. Chem. 2011, 76, 2040. http://dx.doi.org/10.1021/jo102429g

PMid:21370916

(b) Thevenet, D.; Neier, R. Helv. Chim. Acta 2011, 94, 331. http://dx.doi.org/10.1002/hlca.201000333

10. Meier, M. S. in Encyclopedia of Reagents for Organic Synthesis; Paquette, L. A., Ed.; John Wiley \& Sons Inc.: New York, 1995; Vol. 8, pp 5392

11. Ramanathan, M.; Hou, D.-R. Tetrahedron Lett. 2010, 51, 6143. http://dx.doi.org/10.1016/j.tetlet.2010.09.065

12. (a) Gray, G. A. J. Am. Chem. Soc. 1973, 95, 7736.

http://dx.doi.org/10.1021/ja00804a032

(b) Kitahara, T.; Horiguchi, A.; Mori, K. Tetrahedron 1988, 44, 4713; also see supporting information. http://dx.doi.org/10.1016/S0040-4020(01)86174-3

13. Rao, S. V.; Kandula, P. K. Tetrahedron: Asymmetry 2005, 16, 3268. http://dx.doi.org/10.1016/j.tetasy.2005.08.045

14. Castaneda, F.; Aliaga, C.; Acuna, C.; Silva, P.; Bunton, C.A. Phosphorus, Sulfur Silicon Relat. Elem. 2008, 183, 1188. http://dx.doi.org/10.1080/10426500701613030

15. (a) Cordes, E. H.; Bull, H. G. Chem. Rev. 1974, 74, 581. 
http://dx.doi.org/10.1021/cr60291a004

(b)Satchell, D. P. N.; Satchell, R. S. Chem. Soc. Rev. 1990, 19, 55.

http://dx.doi.org/10.1039/cs9901900055

(c) Belarmino, A. T. N.; Froehner, S.; Zanette, D.; Farah, J. P. S.; Bunton, C. A.; Romsted, L. S. J. Org. Chem. 2003, 68, 706.

http://dx.doi.org/10.1021/jo0202987; PMid:12558389

16. The ${ }^{1} \mathrm{H}$ NMR spectra of the prepared compounds $\mathbf{1 8}$ and 19 showed that the cis-isomers were dominant (supporting information). Here, we followed the assignments made by Espenson and Abu-Omar: Zhu, Z.; Espenson, J. H. Organometallics 1997, 16, 3658; Wegenhart, B. L.; Abu-Omar, M. M. Inorg. Chem. 2010, 49, 4741.

17. Shibata, I.; Mitani, I.; Imakuni, A.; Baba, A. Tetrahedron Lett. 2011, 52, 721. http://dx.doi.org/10.1016/j.tetlet.2010.12.009

18. Christol, H. Bull. Soc. Chim. Fr. 1989, 477.

19. Das, P.; McNulty, J. Eur. J. Org. Chem. 2010, 3587. http://dx.doi.org/10.1002/ejoc.201000601

20. (a) Ghosh, A. K.; Gong, G. Chem. Asian J. 2008, 3, 1811. http://dx.doi.org/10.1002/asia.200800164; PMid:18712746 PMCid:3518312

(b) Shiina, I.; Hashizume, M.; Yamai, Y.-s.; Oshiumi, H.; Shimazaki, T.; Takasuna, Y.-j.; Ibuka, R. Chem. Eur. J. 2005, 11, 6601. http://dx.doi.org/10.1002/chem.200500417; PMid:16118824

(c) Wrona, I. E.; Lowe, J. T.; Turbyville, T. J.; Johnson, T. R.; Beignet, J.; Beutler, J. A.; Panek, J. S. J. Org. Chem. 2009, 74, 1897.

http://dx.doi.org/10.1021/jo802269q; PMid:19191575 PMCid:3480235

(d) Breuilles, P.; Oddon, G.; Uguen, D. Tetrahedron Lett. 1997, 38, 6607. http://dx.doi.org/10.1016/S0040-4039(97)01523-2

21. Zhao, Y.-J.; Chng, S.-S.; Loh, T.-P. J. Am. Chem. Soc. 2007, 129, 492. http://dx.doi.org/10.1021/ja067660+; PMid:17227003

22. Smith, B. M.; Graham, A. E. Tetrahedron Lett. 2011, 52, 6281. http://dx.doi.org/10.1016/j.tetlet.2011.09.087

23. Gregg, B. T.; Golden, K. C.; Quinn, J. F. Tetrahedron 2008, 64, 3287. http://dx.doi.org/10.1016/j.tet.2008.01.118

24. Barbasiewicz, M.; Makosza, M. Org. Lett. 2006, 8, 3745. http://dx.doi.org/10.1021/ol0613113; PMid:16898807

25. Briggs, J. R.; Klosin, J.; Whiteker, G. T. Org. Lett. 2005, 7, 495. http://dx.doi.org/10.1021/ol0475008; PMid:15673273

26. Toyota, E.; Sekizaki, H.; Itoh, K.; Tanizawa, K. Chem. Pharm. Bull. 2003, 51, 625. http://dx.doi.org/10.1248/cpb.51.625

27. Anson, C. E.; Creaser, C. S; Malkov, A. V.; Mojovic, L.; Stephenson, G. R. J. Organomet. Chem. 2003, 668, 101. http://dx.doi.org/10.1016/S0022-328X(02)02146-0 
28. Huerta, F. F.; Gómez, C.; Yus, M. Tetrahedron 1999, 55, 4043. http://dx.doi.org/10.1016/S0040-4020(99)00092-7

29. Müller, P.; Nury, P.; Bernardinelli, G. Eur. J. Org. Chem. 2001, 4137. http://dx.doi.org/10.1002/1099-0690(200111)2001:21<4137::AID-EJOC4137>3.0.CO;2-4

30. Huo, C.; Chan, T. H. Adv. Synth. \& Catal. 2009, 351, 1933. http://dx.doi.org/10.1002/adsc.200900102

31. Khupse, R. S.; Erhardt, P. W. J. Nat. Prod. 2007, 70, 1507. http://dx.doi.org/10.1021/np070158y; PMid:17844997

32. Shintou, T.; Mukaiyama, T. J. Am. Chem. Soc. 2004, 126, 7359. http://dx.doi.org/10.1021/ja0487877; PMid:15186175

33. Krasik, P.; Bohemier-Bernard, M.; Yu, Q. Synlett 2005, 854. http://dx.doi.org/10.1055/s-2005-864795

34. Kamble, V. T.; Bandgar, B. T.; Muley, D. B.; Joshi, N. S. J. Mol. Catal. A: Chem. 2007, 268, 70.

http://dx.doi.org/10.1016/j.molcata.2006.12.001 